\title{
Patterns of psychotropic medication use in inpatient and outpatient psychiatric settings in Saudi Arabia
}

\author{
This article was published in the following Dove Press journal: \\ Neuropsychiatric Disease and Treatment \\ 19 April 2016 \\ Number of times this article has been viewed
}

\author{
Fahad D Alosaimi' \\ Abdulhadi Alhabbad ${ }^{2}$ \\ Mohammed F Abalhassan ${ }^{3}$ \\ Ebtihaj O Fallata ${ }^{4}$ \\ Nasser M Alzain 5 \\ Mohammad Zayed Alassiry ${ }^{6}$ \\ Bander Abdullah Haddad ${ }^{7}$ \\ 'Department of Psychiatry, King \\ Saud University, Riyadh, ${ }^{2}$ Department \\ of Psychiatry, Prince Mohammed \\ Medical City, Aljouf, ${ }^{3}$ Department of \\ Medicine, Prince Sattam Bin Abdulaziz \\ University, Al-Kharj, ${ }^{4}$ Department of \\ Psychiatry, Mental Health Hospital, \\ Jeddah, ${ }^{5}$ Department of Psychiatry, \\ Al-Amal Complex for Mental \\ Health, Dammam, ${ }^{6}$ Medical Services \\ Department, Abha Psychiatric \\ Hospital, Abha, ${ }^{7}$ Department \\ of Medicine, King Abdulaziz \\ Medical City, Ministry of National \\ Guard, Riyadh, Saudi Arabia
}

Correspondence: Abdulhadi Alhabbad

Prince Mohammed Medical City, Aljouf,

King Khalid Street, PO Box 88893,

Riyadh 12372, Saudi Arabia

Email abdulhadi.alhabbad@pmmc.med.sa
Objective: To study the pattern of psychotropic medication use and compare this pattern between inpatient and outpatient psychiatric settings in Saudi Arabia.

Method: This cross-sectional observational study was conducted between July 2012 and June 2014 on patients seeking psychiatric advice at major hospitals in five main regions of Saudi Arabia. Male $(n=651)$ and female $(n=594)$ patients who signed the informed consent form and were currently or had been previously using psychotropic medications, irrespective of the patient's type of psychiatric diagnosis and duration of the disease, were included. A total of 1,246 patients were found to be suitable in the inclusion criteria of whom 464 were inpatients while 782 were outpatients.

Results: Several studied demographic factors have shown that compared with outpatients, inpatients were more likely to be male $(P=0.004)$, unmarried $(P<0.001)$, have less number of children ( $1-3 ; P=0.002)$, unemployed $(P=0.001)$, have a lower family income $(<3,000 \mathrm{SR}$; $P<0.001)$, live in rural communities $(P<0.001)$, have a lower body mass index $(P=0.001)$, and are smokers $(P<0.001)$; however, there were no differences with regard to age or educational levels. The current frequency of use of psychotropic medications in overall patients was antipsychotics (76.6\%), antidepressants (41.4\%), mood stabilizers $(27.9 \%)$, and antianxiety (6.2\%). However, compared to outpatients, the current use of medications for inpatients was more frequent $(93.8 \% \mathrm{vs} 89.9 \%, P=0.019)$ with inpatients more likely to be treated with multiple medications (2.1 vs 1.8 medications). A similar trend was observed in the case of antipsychotics, high potency first-generation antipsychotics, second-generation antipsychotics, mood stabilizers, and antianxiety medicines where inpatients were more frequently treated with these medications for all psychiatric diagnoses when compared with outpatients. On the contrary, in the case of antidepressant treatment, an opposite trend was observed with more number of treated outpatients in comparison to inpatients. Among elderly patients, $75.9 \%$ received antipsychotics, mainly second-generation formulations (67.2\%), whereas only $41 \%$ received antidepressants and $13.8 \%$ received mood stabilizers.

Conclusion: Based upon the present study data, it is concluded that among all the psychotropic medications, antipsychotics were heavily used and the frequency was found to be significantly high in the case of inpatients compared with outpatients. Such a practice may lead to multiple negative consequences among the Saudi psychiatric patient population. Further, extensive use of sodium valproate in the case of bipolar disorder, and also among females either in childbearing age or during pregnancy is also the cause of concern and warrants logical use. Overall, this study may help in assessing the burden of psychiatric illness within specific patient demographics and might be effectively used to strategically plan health resources allocation, generate new treatment hypothesis, or be used as a source of evidence that could further integrate other observational studies.

Keywords: psychotropic, inpatient, outpatient, antipsychotics, antidepressants, mood stabilizers, Saudi Arabia 


\section{Introduction}

Psychotropic medications, such as phenothiazine, thioxanthene, or butyrophenone-type neuroleptics, lithium salts, tricyclic antidepressants, irreversible monoamine oxidase inhibitors, and benzodiazepines, have been used for more than 60 years in clinical practices. Most of the new generation of psychotropics have appeared after 1985 and, even more recently, atypical antipsychotics, antiepileptics as mood stabilizers, and antidementia medications. Compared with the old medications, the newer medications have similar efficacies but better tolerability and fewer side effects. ${ }^{1,2}$

Many international studies have directly or indirectly noted how clinical practice and actual psychotropic prescribing practices in both hospital and outpatient settings do not always agree with internationally recognized guidelines. ${ }^{1-4}$ A proper evaluation of the difference between actual practice and a theoretical, gold standard-oriented approach may help to avoid several erroneous trends that may negatively affect patient outcomes and other pharmacoeconomic issues. For example, some international studies have reported an increase in the practice of polypharmacy, that is, the use of four or more medications specially by the elderly population, ${ }^{5}$ and the prescription of new and expensive medications and low-dose and off-label drugs to women and children. ${ }^{4}$ Most of the studies that evaluated use of psychotropic drugs have reported a high rate of prescriptions for multiple drugs, which usually occurs when there was no improvement in clinical symptoms. ${ }^{2}$ A UK survey found that more than $50 \%$ of hospitalized patients were receiving more than one type of antipsychotic medication. ${ }^{6}$

Although hospitalization is often preferred in many major psychiatric diseases, such as schizophrenia or bipolar disorder, treatment as outpatients is also employed to maintain community ties and a patient's autonomy, ${ }^{7}$ thereby resulting in satisfactory treatment results while still reducing overall costs. ${ }^{8}$ Outpatient psychiatric services often complement inpatient ones and limit the excessive use of mental health services; nonetheless, patient populations for whom substitution is possible should be accurately analyzed to avoid an overall reduction of care for patients who fail to follow-up with their treatment. ${ }^{9,10}$

The overall situation of mental health services and prescribing practices in Saudi Arabia has been previously investigated in other studies, but more data are still needed to successfully assess the trends and prescribing habits and to gain a broader view. ${ }^{11}$ Studies with a larger scope have focused more on the mental health system's general situation rather than on a detailed view of the current trends for inpatient and outpatient psychotropic medication prescriptions, ${ }^{12}$ whereas other studies smaller in scope have only analyzed local data. ${ }^{13}$ The objective of the current study was to study the pattern of psychotropic medication use and compare the frequency of prescribing psychotropic medications between inpatient and outpatient settings in Saudi Arabia.

\section{Methods}

\section{Setting}

The current study was conducted among patients seeking psychiatric advice at major hospitals in Saudi Arabia. The patients were recruited from a number of hospitals located in the Central, Eastern, Western, Northern, and Southern regions of Saudi Arabia. The hospitals included in the study were King Khalid University Hospital in Riyadh and Zulfi General Hospital (Central region), Jeddah Mental Health Hospital (Western region), Al Amal Complex for Mental Health - Dammam (Eastern region), Aljouf Mental Health Hospital (Northern region), and Abha Mental Health Hospital (Southern region). King Khalid University Hospital is a university-affiliated governmental hospital, whereas the other hospitals are government-funded hospitals under the authority of the Ministry of Health. All of the hospitals included in this study provide free psychiatric inpatient and outpatient health care services.

\section{Study design}

A cross-sectional observational study was conducted between July 2012 and June 2014. The study obtained all of the required ethical approvals from the Institutional Review Board at the Faculty of Medicine at King Saud University in Riyadh and administrative approvals from the respective hospitals. The study was funded by the National Plan for Science and Technology Strategic Grants Program.

\section{Population}

Male and female patients seeking psychiatric help from the included hospitals during the study period were asked to join the study. Those who signed the informed consent form and were currently or had been previously using psychotropic medications, irrespective of the patient's type of psychiatric diagnosis and the duration of the disease, were included.

\section{Data collection}

A mini-interview form was developed, which included sociodemographic characteristics, detailed current and previous psychotropic medication use (including the name of the medication, dose, and duration), and current psychiatric diagnoses. The information was mainly obtained by 
reviewing each patient's chart. Unclear/missing information was confirmed by interviewing the patient and/or his/her family. Trained psychiatric residents/staff were responsible for chart review and conducting interviews with the patients and/or their families.

\section{Classification of psychotropic medications}

Both the individual psychotropic medications and their pharmacologic groups were used in the analysis. These medications and groups include antidepressants (selective serotonin reuptake inhibitors, tricyclics, and others), antipsychotics (low potency first-generation and high potency first- and second-generation), antianxiety medications, and mood stabilizers.

\section{Classification of psychiatric diagnosis}

For the purpose of data analysis, the psychiatric diagnosis of the studied patients was classified under seven categories. "Primary psychotic disorders" included schizophrenia, schizoaffective disorder, delusional disorder, and brief psychotic disorder. "Primary bipolar disorders" included bipolar disorder and its features. "Primary depressive disorders" included major depressive disorder and dysthymic disorder. "Primary anxiety disorders" included generalized anxiety disorder, obsessive compulsive disorder, social anxiety disorder, specific phobias, panic disorder, post-traumatic stress disorder, and acute stress disorder. "Personality disorders" included mixed personality disorder, paranoid personality disorder, antisocial personality disorder, and borderline personality disorder. "Secondary psychiatric disorders" included psychotic disorder due to another medical condition, depression due to another medical condition, dementia, substance use disorder, and substance-induced depressive disorder. "Other disorders" included somatic symptom disorder, mental retardation, conversion disorder, attention deficit hyperactivity disorder, dissociative disorder, primary insomnia, adjustment disorder, enuresis disorder, trichotillomania, and anorexia nervosa.

\section{Statistical analysis}

The data are presented in frequencies and percentages for categorical data and mean values and standard deviations for continuous data. Individual psychiatric diagnosis and psychotropic medications were categorized into standard groups. Significant differences between inpatient and outpatient settings as they pertained to sociodemographics, psychotropic medication use, and psychiatric diagnosis were tested using the chi-square test or Fisher's exact test (as appropriate) for categorical data and the Student's $t$-test for continuous data. All $P$-values were two-tailed, and a $P$-value $<0.05$ was considered a significant value. SPSS software (release 20.0, SPSS Inc., Chicago, IL, USA) was used for the statistical analysis.

\section{Results}

A total of 1,246 patients (464 inpatients and 782 outpatients) were included in the current analysis.

\section{Demographic characteristics}

Table 1 demonstrates the demographic characteristics of inpatients and outpatients studied. In terms of age, both types of patients were categorized into three categories, that is, $<40,40-60$, and $>60$ years. Inpatients and outpatients were found to show similar trend with the average majority (58.9\%) lying below the age of 40 followed by the age of 40-60 (35.9\%) and $>60(5.2 \%)$. Similarly, no major sex difference was noticed with an average of $52.3 \%$ males and $47.7 \%$ females. Approximately $45.6 \%$ of the patients were married, and 54.4\% were unmarried: single (44.1\%), divorced $(9.2 \%)$, or widowed $(1.1 \%)$. However, significant difference $(P<0.001)$ was obtained in this case where inpatients have a majority of single and divorced persons while outpatients have a maximum number of married and widowed persons.

Further, $\sim 75.9 \%$ of the patients had children, in which the percentage of inpatients was more who possess one to three children in comparison with outpatients while the opposite was obtained where the number of children exceeds three.

All inpatients and outpatients were also recorded for their education level as illiterates, possess secondary education or less, and university level. No noticeable differences were observed in any of the education levels when compared between inpatients and outpatients, with an average majority (65.0\%) falling in the category of less than secondary education.

When analyzed for their job status, partial variations were observed in patients who were in government job and unemployed persons. While more number of outpatients was recorded in the former condition, more number of inpatients was found in the latter status. However, overall among all the job status (private, government, business, or unemployed), the highest average ( $71.4 \%$ ) belongs to unemployed patients. Approximately $61.9 \%$ of the total patients had a moderately low family monthly income $(6,000$ Saudi Riyal [SR] or less). Marked differences $(P<0.001)$ were found with the number of inpatients comparatively more in low 
Table I Demographic characteristics by type of patient setting $(\mathrm{N}=\mathrm{I}, 246)$

\begin{tabular}{|c|c|c|c|c|}
\hline Characteristic & $\begin{array}{l}\text { Inpatient } \\
(\mathrm{N}=464)\end{array}$ & $\begin{array}{l}\text { Outpatient } \\
(\mathrm{N}=\mathbf{7 8 2})\end{array}$ & $\begin{array}{l}\text { Total } \\
(\mathrm{N}=1,246)\end{array}$ & $P$-value \\
\hline \multicolumn{5}{|l|}{ Age, years } \\
\hline Mean \pm SD & $37.6 \pm 12.1$ & $38.6 \pm 13.6$ & $38.2 \pm 13.0$ & 0.191 \\
\hline$<40$ & $282(61.0 \%)$ & 448 (57.7\%) & $730(58.9 \%)$ & 0.305 \\
\hline $40-60$ & $16 \mid(34.8 \%)$ & $284(36.6 \%)$ & 445 (35.9\%) & \\
\hline$>60$ & $19(4.1 \%)$ & $45(5.8 \%)$ & $64(5.2 \%)$ & \\
\hline \multicolumn{5}{|l|}{ Sex } \\
\hline Male & 267 (57.5\%) & $384(49.2 \%)$ & 65 I (52.3\%) & 0.004 \\
\hline Female & 197 (42.5\%) & 397 (50.8\%) & 594 (47.7\%) & \\
\hline \multicolumn{5}{|l|}{ Marital status } \\
\hline Married & 119 (27.9\%) & $4 \mid 8(55.7 \%)$ & $537(45.6 \%)$ & $<0.001$ \\
\hline Single & 245 (57.4\%) & 275 (36.6\%) & $520(44.1 \%)$ & \\
\hline Divorced & 61 (14.3\%) & $47(6.3 \%)$ & $108(9.2 \%)$ & \\
\hline Widowed & $2(0.5 \%)$ & II (I.5\%) & $13(1.1 \%)$ & \\
\hline \multicolumn{5}{|l|}{ Number of children } \\
\hline Mean \pm SD & $3.1 \pm 2.9$ & $3.6 \pm 3.2$ & $3.5 \pm 3.1$ & 0.077 \\
\hline None & 39 (21.7\%) & $119(25.1 \%)$ & $158(24.1 \%)$ & 0.002 \\
\hline I-3 children & 79 (43.9\%) & I 40 (29.5\%) & $219(33.4 \%)$ & \\
\hline$>3$ children & $62(34.4 \%)$ & $216(45.5 \%)$ & 278 (42.4\%) & \\
\hline \multicolumn{5}{|l|}{ Educational level } \\
\hline Illiterate & $96(21.8 \%)$ & 165 (21.4\%) & $26 \mathrm{I}(2 \mathrm{I} .5 \%)$ & 0.261 \\
\hline Secondary or less & 295 (66.9\%) & $493(63.9 \%)$ & $788(65.0 \%)$ & \\
\hline University/others & $50(11.3 \%)$ & $113(14.7 \%)$ & $163(13.4 \%)$ & \\
\hline \multicolumn{5}{|l|}{ Work type } \\
\hline Private & 35 (7.8\%) & $64(8.3 \%)$ & $99(8.1 \%)$ & 0.001 \\
\hline Governmental & 53 (11.8\%) & 155 (20.2\%) & $208(17.1 \%)$ & \\
\hline Own business & $13(2.9 \%)$ & $28(3.7 \%)$ & $4 \mathrm{I}(3.4 \%)$ & \\
\hline Unemployed & $347(77.5 \%)$ & $520(67.8 \%)$ & 867 (7I.4\%) & \\
\hline \multicolumn{5}{|l|}{ Monthly family income (Saudi Riyal) } \\
\hline Low income $(\leq 3,000)$ & $162(37.2 \%)$ & $194(25.5 \%)$ & $356(29.7 \%)$ & $<0.001$ \\
\hline Lower limit of moderate income $(3,00 \mathrm{I}-6,000)$ & $130(29.9 \%)$ & $256(33.6 \%)$ & $386(32.2 \%)$ & \\
\hline Upper limit of moderate income $(6,001-9,000)$ & $94(21.6 \%)$ & $151(19.8 \%)$ & $245(20.5 \%)$ & \\
\hline High income $(>9,000)$ & 49 (II.3\%) & $161(21.1 \%)$ & $210(17.5 \%)$ & \\
\hline \multicolumn{5}{|l|}{ Residency } \\
\hline City & $37 \mid(80.8 \%)$ & $630(80.9 \%)$ & $\mathrm{I}, 00 \mathrm{I}(80.9 \%)$ & $<0.001$ \\
\hline Village & 88 (19.2\%) & $121(15.5 \%)$ & $209(16.9 \%)$ & \\
\hline Desert & $0(0.0 \%)$ & $28(3.6 \%)$ & $28(2.3 \%)$ & \\
\hline \multicolumn{5}{|l|}{ Body mass index } \\
\hline Mean \pm SD & $27.7 \pm 7.6$ & $29.2 \pm 7.5$ & $28.6 \pm 7.6$ & 0.001 \\
\hline Underweight & $36(8.3 \%)$ & $34(4.4 \%)$ & $70(5.8 \%)$ & 0.001 \\
\hline Normal & $13 \mid(30.3 \%)$ & 195 (25.2\%) & $326(27.0 \%)$ & \\
\hline Overweight & $131(30.3 \%)$ & $225(29.1 \%)$ & $356(29.5 \%)$ & \\
\hline Obese & 135 (3|.2\%) & $319(41.3 \%)$ & $454(37.6 \%)$ & \\
\hline \multicolumn{5}{|l|}{ Smoking status } \\
\hline Current & $|8|(40.0 \%)$ & $234(30.0 \%)$ & 415 (33.7\%) & $<0.001$ \\
\hline Past & 70 (15.5\%) & $78(10.0 \%)$ & $148(12.0 \%)$ & \\
\hline Never & $202(44.6 \%)$ & 467 (59.9\%) & 669 (54.3\%) & \\
\hline
\end{tabular}

Notes: Bold indicates statistically significant results $(P<0.05)$. A BMl below $18.5 \mathrm{~kg} / 2$ is considered underweight. $\mathrm{A} \mathrm{BMl}$ of 18.5 to $24.9 \mathrm{~kg} / \mathrm{m}^{2}$ is considered healthy (normal). A BMI of 25 to $29.9 \mathrm{~kg} / \mathrm{m}^{2}$ is considered overweight. A BMl of $30 \mathrm{~kg} / \mathrm{m}^{2}$ or higher is considered obese.

Abbreviation: SD, standard deviation.

income category $(<3,000 \mathrm{SR})$ and number of outpatients in high income category ( $>9,000 \mathrm{SR})$. The majority $(80.9 \%)$ of the total patients reside in urban communities, with no major differences between the inpatient and outpatient count. The average body mass index was $28.6 \pm 7.6$, and $67.1 \%$ of patients were either overweight or obese. Approximately
$45.7 \%$ of patients were either current $(33.7 \%)$ or previous $(12.0 \%)$ smokers.

Overall, compared with outpatients, inpatients were more likely to be male $(P=0.004)$, unmarried $(P<0.001)$, have less number of children $(1-3 ; P=0.002)$, unemployed $(P=0.001)$, have a lower family income $(<3,000 \mathrm{SR}$; 
$P<0.001)$, live in rural communities $(P<0.001)$, have a lower body mass index $(P=0.001)$, and smokers $(P<0.001)$; however, there were no differences with regard to age or educational levels.

\section{Current and previous use of antipsychotic medications in inpatients and outpatients}

Figure 1 illustrates the overall usage of psychotropic medications in current as well as in previous scenario and the following frequencies were recorded: antipsychotics $(76.6 \%$ and $76.1 \%$, respectively), antidepressants $(41.4 \%$ and $38.5 \%$, respectively), mood stabilizers (27.9\% and $23.5 \%$, respectively), and antianxiety medications ( $6.2 \%$ and $3.6 \%$, respectively). Compared to outpatients, the current use of medications for inpatients was more frequent $(93.8 \%$ vs $89.9 \%, P=0.019)$, whereas their previous medication use was approximately similar $(76.3 \%$ vs $78.1 \%, P=0.452)$. Additionally, inpatients were more likely to currently $(2.1 \pm 0.8$ vs $1.8 \pm 0.7, P<0.001)$ and previously $(2.0 \pm 0.8$ vs $1.7 \pm 0.7, P<0.001)$ use multiple medications compared with outpatients.

The current and previous uses of different psychotropic medications in inpatients and outpatients are shown in Tables 2-4. With very few exceptions, different groups of antipsychotic medications were both currently and previously more frequently used among inpatients compared with outpatients (Table 2). For example, antipsychotic medications were currently used for $94.3 \%$ of inpatients and $65.7 \%$ for outpatients $(P<0.001)$. This trend was similarly observed in the heavily used second-generation group and less commonly used high potency first-generation groups ( $P<0.001$ for all), but not in low potency first-generation group. However, the different groups of antidepressant medications were both currently and previously more frequently used among outpatients compared with inpatients (Table 3). For example, any antidepressant medications were currently used in $54.1 \%$ of outpatient settings and $20.9 \%$ of inpatient settings $(P<0.001)$. This trend was similarly observed within the commonly used groups, such as selective serotonin reuptake inhibitors, and with the less commonly used tricyclics and other types of antidepressants $(P<0.001$ for all). Current and previous uses of mood stabilizers and antianxiety medications were more frequently observed among inpatients compared with outpatients (Table 4). For example, mood stabilizers were currently used for $40.7 \%$ of inpatients and $20.1 \%$ of outpatients $(P<0.001)$, and antianxiety medications were currently used for $13.3 \%$ of inpatients and $1.8 \%$ of outpatients $(P<0.001)$.

Table 5 shows the current prevalence in the prescription of psychotropic medications according to psychiatric diagnosis in both inpatient and outpatient settings. Antipsychotic medications were heavily used (more than $85 \%$ ) in almost all inpatient psychiatric diagnosis, with the exception of primary anxiety and depressive disorders. Additionally, antipsychotic use was more frequent among inpatients than outpatients for all psychiatric diagnosis, with the exception of personality and secondary psychiatric disorders. Antidepressant medications were heavily used ( $80 \%$ or more) in outpatient settings for primary anxiety disorders, primary depressive disorders, and personality disorders. Additionally, antidepressant use was more common for outpatients than for inpatients, especially for those with primary anxiety disorders and primary bipolar disorders. Mood stabilizers were frequently used for primary bipolar disorders among inpatients and outpatients $(\sim 75 \%)$, and their use was generally higher among inpatients

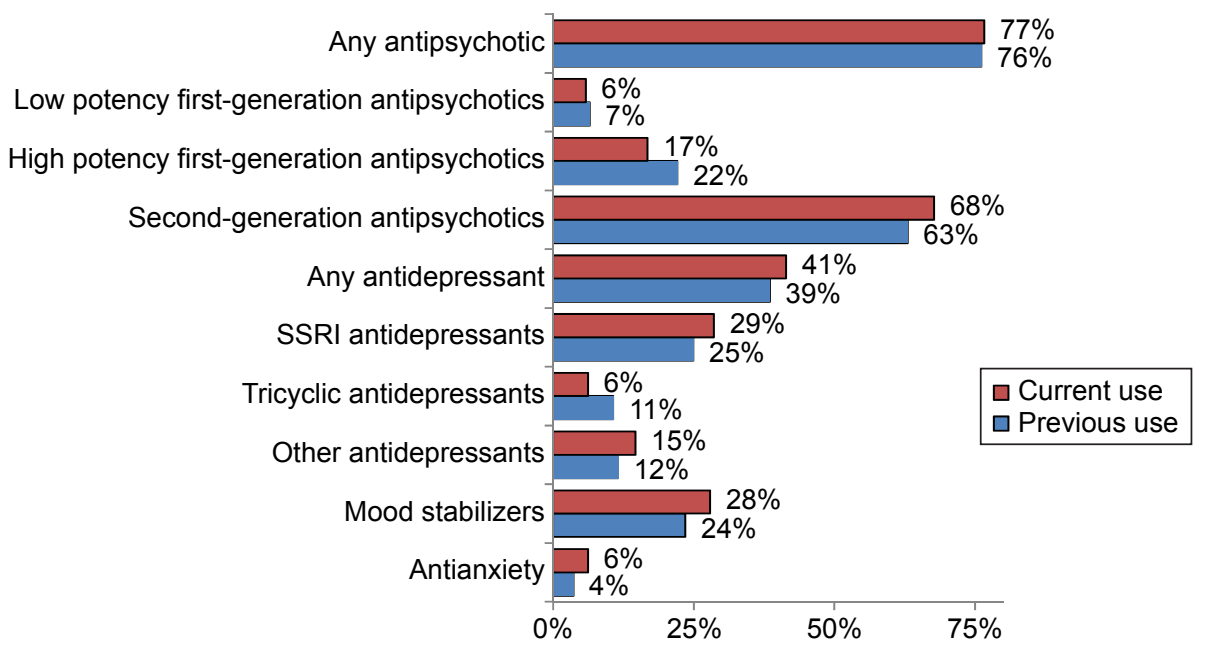

Figure I Frequencies of current and previous uses of different psychotropic medications $(N=I, 246)$. Abbreviation: SSRI, Selective serotonin reuptake inhibitor. 
Table 2 Current and previous uses of antipsychotic medications by type of patient setting $(\mathrm{N}=\mathrm{I}, 246)$

\begin{tabular}{|c|c|c|c|c|c|c|c|c|}
\hline \multirow{2}{*}{$\begin{array}{l}\text { Classes of } \\
\text { antipsychotics used }\end{array}$} & \multicolumn{3}{|l|}{ Current } & \multirow[t]{2}{*}{$P$-value } & \multicolumn{3}{|l|}{ Previous } & \multirow[t]{2}{*}{$P$-value } \\
\hline & Inpatient (\%) & Outpatient (\%) & Total (\%) & & Inpatient (\%) & Outpatient (\%) & Total (\%) & \\
\hline Any antipsychotic & $410(94.3)$ & $462(65.7)$ & $872(76.6)$ & $<0.001$ & $318(89.8)$ & $416(68.1)$ & $734(76.1)$ & $<0.001$ \\
\hline Low potency & $26(6.0)$ & $40(5.7)$ & $66(5.8)$ & 0.84 & $19(5.4)$ & $44(7.2)$ & $63(6.5)$ & 0.266 \\
\hline \multicolumn{9}{|l|}{ first-generation } \\
\hline Chlorpromazine & $25(5.7)$ & $32(4.6)$ & $57(5.0)$ & 0.369 & $17(4.8)$ & $31(5.1)$ & $48(5.0)$ & 0.852 \\
\hline Thioridazine & $0(0.0)$ & $0(0.0)$ & $0(0.0)$ & - & $2(0.6)$ & $I(0.2)$ & $3(0.3)$ & 0.558 \\
\hline Sulpiride & $\mathrm{I}(0.2)$ & $8(1.1)$ & $9(0.8)$ & 0.165 & $2(0.6)$ & $13(2.1)$ & $15(1.6)$ & 0.059 \\
\hline High potency & $113(26.0)$ & $78(11.1)$ & $191(16.8)$ & $<0.00$ I & $100(28.2)$ & $113(18.5)$ & $213(22.1)$ & $<\mathbf{0 . 0 0 1}$ \\
\hline \multicolumn{9}{|l|}{ first-generation } \\
\hline Haloperidol & $87(20.0)$ & $44(6.3)$ & $|3|(|| .5)$ & $<0.001$ & $80(22.6)$ & $60(9.8)$ & $140(14.5)$ & $<\mathbf{0 . 0 0 1}$ \\
\hline Trifluoperazine & $8(1.8)$ & $20(2.8)$ & $28(2.5)$ & 0.287 & $13(3.7)$ & $48(7.9)$ & $61(6.3)$ & 0.010 \\
\hline Fluphenazine & $15(3.4)$ & $4(0.6)$ & $19(1.7)$ & $<0.001$ & $7(2.0)$ & $8(1.3)$ & $15(1.6)$ & 0.419 \\
\hline Flupentixol & $6(1.4)$ & $7(1.0)$ & $13(1.1)$ & 0.575 & $9(2.5)$ & $6(1.0)$ & $15(1.6)$ & 0.059 \\
\hline Zuclopenthixol & $0(0.0)$ & $6(0.9)$ & $6(0.5)$ & 0.088 & $3(0.8)$ & $0(0.0)$ & $3(0.3)$ & 0.049 \\
\hline Second-generation & $365(83.9)$ & $405(57.6)$ & 770 (67.7) & $<0.001$ & $277(78.2)$ & 331 (54.2) & $608(63.0)$ & $<0.001$ \\
\hline Risperidone & $159(36.6)$ & $165(23.5)$ & $324(28.5)$ & $<0.001$ & $140(39.5)$ & $159(26.0)$ & $299(31.0)$ & $<0.001$ \\
\hline Olanzapine & $137(3 \mid .5)$ & $133(18.9)$ & $270(23.7)$ & $<0.00$ I & $117(33.1)$ & $110(18.0)$ & $227(23.5)$ & $<\mathbf{0 . 0 0 1}$ \\
\hline Quetiapine & $68(15.6)$ & $104(14.8)$ & $172(15.1)$ & 0.701 & $58(16.4)$ & 7I (II.6) & $129(13.4)$ & 0.036 \\
\hline Aripiprazole & $52(12.0)$ & $55(7.8)$ & $107(9.4)$ & 0.020 & $16(4.5)$ & $27(4.4)$ & $43(4.5)$ & 0.942 \\
\hline Paliperidone & $29(6.7)$ & $20(2.8)$ & $49(4.3)$ & 0.002 & $10(2.8)$ & $10(1.6)$ & $20(2.1)$ & 0.212 \\
\hline Clozapine & $23(5.3)$ & $12(1.7)$ & $35(3.1)$ & 0.001 & $12(3.4)$ & II (I.8) & $23(2.4)$ & 0.119 \\
\hline Amisulpride & $9(2.1)$ & $5(0.7)$ & $14(1.2)$ & 0.043 & $3(0.8)$ & $5(0.8)$ & $8(0.8)$ & 1.000 \\
\hline
\end{tabular}

Note: Bold indicates statistically significant results $(P<0.05)$.

than among outpatients. Antianxiety medications were largely administered (14\%-27\%) for primary bipolar disorders, primary anxiety disorders, and personality disorders, mainly among inpatients.

Among all of the participants, lithium was only used by $2.6 \%$ of participants, compared to $26.7 \%$ of participants using various anticonvulsants (sodium valproate 18.6\%, carbamazepine $5.8 \%$, and lamotrigine $2.3 \%$ ). Specifically, among women of childbearing age (18-45 years), sodium valproate was the most used mood-stabilizing agent $(20.8 \%)$, whereas lithium was used only by 3.6\%. Moreover, during pregnancy, there is a trend of preferring sodium valproate

Table 3 Current and previous uses of antidepressant medications by type of patient setting $(\mathrm{N}=\mathrm{I}, 246)$

\begin{tabular}{|c|c|c|c|c|c|c|c|c|}
\hline \multirow{2}{*}{$\begin{array}{l}\text { Classes of } \\
\text { antidepressants used }\end{array}$} & \multicolumn{3}{|l|}{ Current } & \multirow[t]{2}{*}{$P$-value } & \multicolumn{3}{|l|}{ Previous } & \multirow[t]{2}{*}{$P$-value } \\
\hline & Inpatient (\%) & Outpatient (\%) & Total (\%) & & Inpatient (\%) & Outpatient (\%) & Total (\%) & \\
\hline Any antidepressant & $91(20.9)$ & $380(54.1)$ & $47 I(4 I .4)$ & $<\mathbf{0 . 0 0 1}$ & $74(20.9)$ & $298(48.8)$ & $372(38.5)$ & $<0.001$ \\
\hline SSRI antidepressants & $61(14.0)$ & $265(37.7)$ & $326(28.6)$ & $<\mathbf{0 . 0 0 1}$ & $44(12.4)$ & $196(32.1)$ & $240(24.9)$ & $<0.001$ \\
\hline Escitalopram & $22(5.1)$ & $107(15.2)$ & $129(11.3)$ & $<\mathbf{0 . 0 0 1}$ & $13(3.7)$ & $63(10.3)$ & $76(7.9)$ & $<0.001$ \\
\hline Fluoxetine & $15(3.4)$ & $67(9.5)$ & $82(7.2)$ & $<\mathbf{0 . 0 0 I}$ & $16(4.5)$ & $56(9.2)$ & $72(7.5)$ & 0.008 \\
\hline Citalopram & $3(0.7)$ & $39(5.5)$ & $42(3.7)$ & $<\mathbf{0 . 0 0 1}$ & $3(0.8)$ & $44(7.2)$ & $47(4.9)$ & $<\mathbf{0 . 0 0 1}$ \\
\hline Paroxetine & $8(1.8)$ & $28(4.0)$ & $36(3.2)$ & 0.045 & $7(2.0)$ & $19(3.1)$ & $26(2.7)$ & 0.295 \\
\hline Fluvoxamine & $8(1.8)$ & $16(2.3)$ & $24(2.1)$ & 0.618 & $7(2.0)$ & $18(2.9)$ & $25(2.6)$ & 0.361 \\
\hline Sertraline & $5(1.1)$ & $14(2.0)$ & $19(1.7)$ & $0.28 \mathrm{I}$ & $2(0.6)$ & $13(2.1)$ & $15(1.6)$ & 0.059 \\
\hline Tricyclic antidepressants & $8(1.8)$ & $62(8.8)$ & $70(6.2)$ & $<\mathbf{0 . 0 0 1}$ & $15(4.2)$ & $88(14.4)$ & $103(10.7)$ & $<\mathbf{0 . 0 0 1}$ \\
\hline Amitriptyline & $7(1.6)$ & $51(7.3)$ & $58(5.1)$ & $<\mathbf{0 . 0 0 I}$ & $13(3.7)$ & $74(12.1)$ & $87(9.0)$ & $<0.001$ \\
\hline Imipramine & $0(0.0)$ & $6(0.9)$ & $6(0.5)$ & 0.088 & $0(0.0)$ & $8(1.3)$ & $8(0.8)$ & 0.057 \\
\hline Clomipramine & $\mathrm{I}(0.2)$ & $4(0.6)$ & $5(0.4)$ & 0.655 & $\mathrm{I}(0.3)$ & $7(1.1)$ & $8(0.8)$ & 0.270 \\
\hline Maprotiline & $0(0.0)$ & $I(0.1)$ & $\mathrm{I}(0.1)$ & 1.000 & $\mathrm{I}(0.3)$ & $\mathrm{I}(0.2)$ & $2(0.2)$ & 1.000 \\
\hline Other antidepressants & $34(7.8)$ & $132(18.8)$ & $166(14.6)$ & $<\mathbf{0 . 0 0 1}$ & $23(6.5)$ & $88(14.4)$ & III (II.5) & $<\mathbf{0 . 0 0 1}$ \\
\hline Mirtazapine & $16(3.7)$ & $81(11.5)$ & $97(8.5)$ & $<0.001$ & $13(3.7)$ & $50(8.2)$ & $63(6.5)$ & 0.006 \\
\hline Venlafaxine & $16(3.7)$ & $42(6.0)$ & $58(5.1)$ & 0.087 & $8(2.3)$ & $35(5.7)$ & $43(4.5)$ & 0.012 \\
\hline Agomelatine & I $(0.2)$ & $7(1.0)$ & $8(0.7)$ & 0.164 & $2(0.6)$ & $0(0.0)$ & $2(0.2)$ & 0.134 \\
\hline Duloxetine & $\mathrm{I}(0.2)$ & $5(0.7)$ & $6(0.5)$ & 0.416 & $0(0.0)$ & $6(1.0)$ & $6(0.6)$ & 0.091 \\
\hline Desvenlafaxine & $0(0.0)$ & $2(0.3)$ & $2(0.2)$ & 0.527 & $0(0.0)$ & $\mathrm{I}(0.2)$ & $\mathrm{I}(0.1)$ & 1.000 \\
\hline Trazodone & $0(0.0)$ & $\mathrm{I}(0.1)$ & $\mathrm{I}(0.1)$ & 1.000 & $\mathrm{I}(0.3)$ & $\mathrm{I}(0.2)$ & $2(0.2)$ & 1.000 \\
\hline Maprotiline & $0(0.0)$ & $\mathrm{I}(0.1)$ & $\mathrm{I}(0.1)$ & 1.000 & $\mathrm{I}(0.3)$ & $\mathrm{I}(0.2)$ & $2(0.2)$ & 1.000 \\
\hline
\end{tabular}

Note: Bold indicates statistically significant results $(P<0.05)$.

Abbreviation: SSRI, Selective serotonin reuptake inhibitor. 
Table 4 Current and previous uses of other psychotropic medications by type of patient setting $(\mathrm{N}=\mid, 246)$

\begin{tabular}{|c|c|c|c|c|c|c|c|c|}
\hline \multirow{2}{*}{$\begin{array}{l}\text { Other } \\
\text { psychotropics used }\end{array}$} & \multicolumn{3}{|l|}{ Current } & \multirow[t]{2}{*}{$P$-value } & \multicolumn{3}{|l|}{ Previous } & \multirow[t]{2}{*}{$P$-value } \\
\hline & Inpatient (\%) & Outpatient (\%) & Total (\%) & & Inpatient (\%) & Outpatient (\%) & Total (\%) & \\
\hline Mood stabilizers & $177(40.7)$ & $14 \mid(20.1)$ & 318 (27.9) & $<0.001$ & $129(36.4)$ & $98(16.0)$ & $227(23.5)$ & $<\mathbf{0 . 0 0 1}$ \\
\hline Valproate & $123(28.3)$ & $88(12.5)$ & $211(18.5)$ & $<0.001$ & $85(24.0)$ & $47(7.7)$ & $132(13.7)$ & $<0.001$ \\
\hline Carbamazepine & $36(8.3)$ & $31(4.4)$ & $67(5.9)$ & 0.007 & $30(8.5)$ & $33(5.4)$ & $63(6.5)$ & 0.062 \\
\hline Lithium & $16(3.7)$ & $13(1.8)$ & $29(2.5)$ & 0.057 & II (3.I) & $15(2.5)$ & $26(2.7)$ & 0.546 \\
\hline Lamotrigine & $10(2.3)$ & $16(2.3)$ & $26(2.3)$ & 0.980 & $12(3.4)$ & $8(1.3)$ & $20(2.1)$ & 0.029 \\
\hline Antianxiety & $58(13.3)$ & $13(1.8)$ & 71 (6.2) & $<0.001$ & $29(8.2)$ & $6(1.0)$ & $35(3.6)$ & $<\mathbf{0 . 0 0 1}$ \\
\hline Clonazepam & $32(7.4)$ & $8(1.1)$ & $40(3.5)$ & $<0.001$ & $12(3.4)$ & $2(0.3)$ & $14(1.5)$ & $<0.001$ \\
\hline Lorazepam & $23(5.3)$ & $\mathrm{I}(0.1)$ & $24(2.1)$ & $<0.001$ & $18(5.1)$ & $2(0.3)$ & $20(2.1)$ & $<0.001$ \\
\hline Alprazolam & $2(0.5)$ & $3(0.4)$ & $5(0.4)$ & 1.000 & $0(0.0)$ & $2(0.3)$ & $2(0.2)$ & 0.535 \\
\hline Diazepam & $\mathrm{I}(0.2)$ & $\mathrm{I}(0.1)$ & $2(0.2)$ & 1.000 & $0(0.0)$ & $0(0.0)$ & $0(0.0)$ & - \\
\hline Others & $0(0.0)$ & $2(0.3)$ & $2(0.2)$ & 0.527 & $\mathrm{I}(0.3)$ & $0(0.0)$ & I $(0.1)$ & 0.367 \\
\hline Atomoxetine & $0(0.0)$ & $\mathrm{I}(0.1)$ & $\mathrm{I}(0.1)$ & 1.000 & $0(0.0)$ & $0(0.0)$ & $0(0.0)$ & - \\
\hline Methylphenidate & $0(0.0)$ & $\mathrm{I}(0.1)$ & I $(0.1)$ & 1.000 & $\mathrm{I}(0.3)$ & $0(0.0)$ & I $(0.1)$ & 0.367 \\
\hline
\end{tabular}

Notes: Bold indicates statistically significant results $(P<0.05)$. Dash indicates data not applicable.

as a mood-stabilizing agent $(7.7 \%)$ over lithium $(0 \%)$, although the small sample size of the pregnant participants (15 participants, $2.5 \%$ ) makes it difficult to attain statistically significant results.

Among elderly patients (older than 60 years), 75.9\% received antipsychotics, mainly second-generation drugs $(67.2 \%)$, whereas only $41 \%$ received antidepressants and $13.8 \%$ received mood stabilizers.

\section{Discussion}

The present study represented and analyzed data about the use of psychotropic medications in inpatients and outpatients from six hospitals in Saudi Arabia. These data clearly indicate that a large percentage of the total inpatient and outpatient participants (77\%, Figure 1) received at least one antipsychotic drug while other drugs were prescribed in much smaller percentages, including antidepressants $(41 \%)$, mood stabilizers $(28 \%)$, and antianxiety drugs $(6 \%)$. Further, it was also noticed that in comparison to outpatients (65.7\%), almost all hospitalized patients (94.3\%) were more often treated with at least one antipsychotic medication. On the contrary, a much larger percentage of outpatients were treated with antidepressants of any kind compared with inpatients (54.1\% vs $20.9 \%$, Table 3$)$. The use of antipsychotic medications for the treatment of several major psychiatric diseases, including depression, ${ }^{5}$ is becoming common practice. The present data suggest that inpatients suffering from depression are more often managed by using psychotropic medications, which may necessitate a high level of care for their appropriate prescription and dosing. ${ }^{3}$ It is to be noticed that treatment with antipsychotic medications (alone or in combination) showed a better response rate only if the patient suffers from major psychotic depression, ${ }^{6}$ and these drugs may possess severe adverse side effects that could cause negative outcomes ${ }^{7,8}$ and could increase morbidity and mortality from a range of medical causes. ${ }^{9}$ Considering the overuse of psychotropic medications as also indicated by the present study data, it is strongly recommended that great care and attention should be paid while prescribing these medications specifically for the agents which lack adequate scientific evidence. ${ }^{4}$ A similar trend was also found in case of mood stabilizers and antianxiety drugs where frequency of prescription of these drugs was more in inpatients $(40.7 \%$ and $13.3 \%)$ than outpatients $(20.1 \%$ and $1.8 \%$, Table 4$)$.

The present study also noticed that patients on antipsychotic medications in Saudi Arabia possess several risk factors, such as smoking, dyslipidemia, overweight, and obesity. As shown in the demographic characteristics in Table 1, $\sim 45.7 \%$ of the patients were either current $(33.7 \%)$ or previous $(12.0 \%)$ smokers, and $67.1 \%$ of them were either overweight or obese. While these risk factors are reportedly associated with major mental illnesses, such as schizophrenia or bipolar disorder, ${ }^{13,14}$ they also lead to increased susceptibility for progression of diabetes, weight gain, and other metabolic and cardiovascular diseases. ${ }^{11,12}$ Further, association of other demographic factors was also studied for all the participants. The study findings revealed that more than half of the patients (54.4\%, Table 1) are unmarried - single (44.1\%), divorced, $(9.2 \%)$, or widowed $(1.1 \%)$, although approximately threequarters of them $(75.9 \%)$ had at least one child. It is known that family support and social interactions play a crucial role in reducing psychotic episodes, and encourage patients to improve their compliance with drug treatments. ${ }^{15,16}$ Since a large percentage of Saudi Arabian psychiatric patients live in unstable family environments or alone, a series of health policies focusing on a more attentive follow-up treatment after discharge should be considered as a critical part of treatment. 


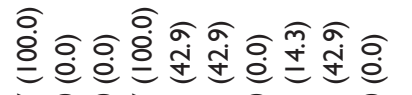

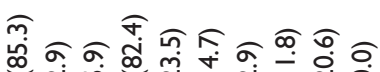

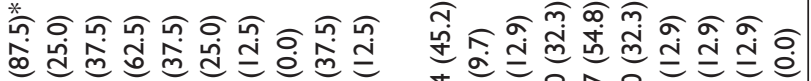

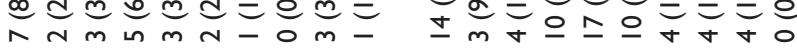

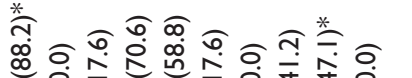

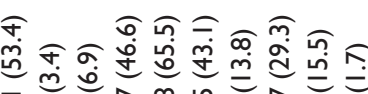

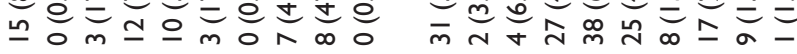

人onmmo-mo

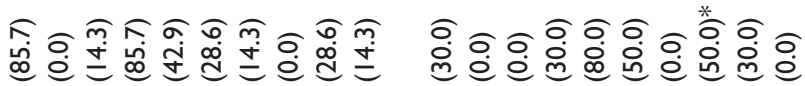

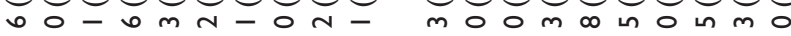

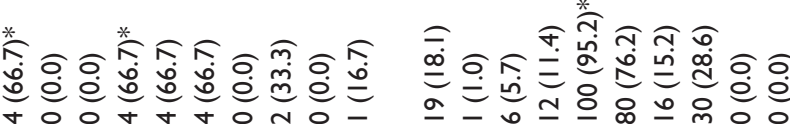

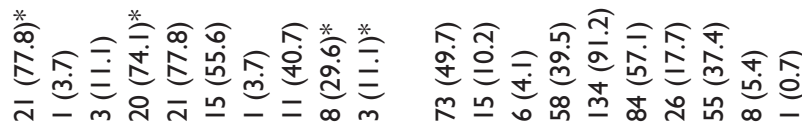

K. $k \sigma$

ลำ

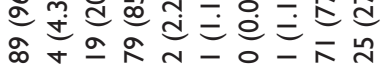

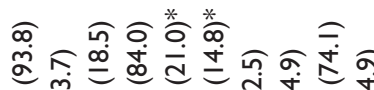
ㄴำ

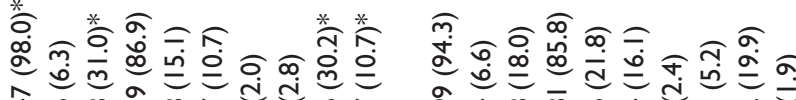

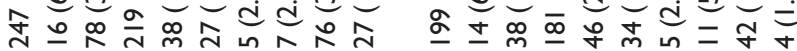

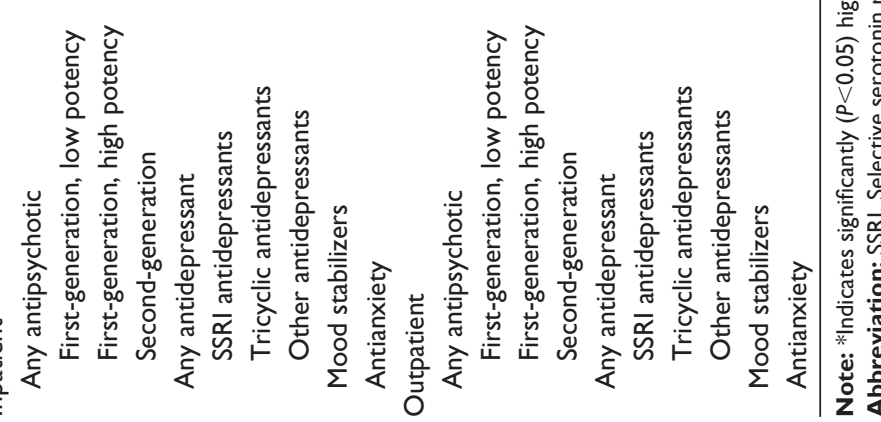


Further, a majority of these psychiatric patients were unemployed (71.4\%), perhaps either due to being illiterate $(21.5 \%)$ or with lowest education $(65.0 \%)$, thereby living in poverty (29.7\% of them had a monthly family income of $<3,000$ SR) (Table 1). It is still not clear whether these factors either contribute to the increased prevalence or outcome of psychiatric disorders; previous findings also reported a similar correlation between poverty and/or social vulnerability with mental illness in other countries..$^{17,18}$

Second-generation antipsychotics are vastly preferred to both high- and low potency first-generation antipsychotics ( $68 \%$ total patients vs $6 \%$ and $17 \%$, Figure 1 and Table 2 ) due to their better safety profiles and improved tolerability. ${ }^{19}$ However, a strong underuse of clozapine can be noted (only 3.1\% of total inpatients and $2.4 \%$ of total outpatients, as shown in Table 2), even though this drug is highly recommended among the other antipsychotics. ${ }^{1}$ Clozapine underuse has also been reported in the USA and the UK, which may be caused by a tendency on the clinician's part to overestimate the relevance and severity of side effects. ${ }^{20-22}$ Further, aggressive marketing of other second-generation antipsychotics from pharmaceutical companies may also be a reason why clozapine use is decreasing in hospitals all over the world, including in Saudi Arabia. ${ }^{23}$ Several studies suggested that detailed marketing campaigns from pharmaceutical companies involved presentation of inaccurate or suppressed data and even more the criteria for mental disorders were in fact stretched to target a vast range of patients. ${ }^{24,25}$ Several pharmaceutical companies adopted aggressive sales strategies, such as, advertising directly to consumers, influencing prescribers, and paying physicians to market to their peers, presenting overly optimistic cost-effectiveness research, providing free drug samples, sponsoring medical education and patient advocacy groups, and publication of biased results, in order to push widespread usage of second-generation antipsychotics. ${ }^{26}$

Our data also confirm that mood stabilizers (especially sodium valproate) are often used in addition to primary therapy with antipsychotics for the treatment of most cases of bipolar disorder (77.2\%, Table 5). Although this trend is positive, as prescribing sodium valproate in combination with antipsychotics provided better outcomes with mania than with antipsychotic medications alone, effectively lowering the antipsychotic medications' dosages and consequently their side effects, ${ }^{27}$ it should also be noted that valproate and carbamazepine are much more frequently used to treat bipolar disorder compared to lithium salt, which should be the current gold standard (343 and 130 total patients for valproate and carbamazepine, respectively, compared to just
55 total patients for lithium). ${ }^{28}$ Other international studies have shown similar trends, suggesting that although lithium remains the gold standard of treatment, newer agents may sometimes be considered advances in treatment in local clinical practice and habits. ${ }^{28,29}$ Moreover, among women of childbearing age and even probably among pregnant women, sodium valproate was overused compared to lithium. Similar practices have also been noted in the $\mathrm{UK}^{30}$ and the USA. ${ }^{31}$ This practice is contrary to recent guidelines that recommend lithium use in this group, granting it higher efficacy data and a safer reproductive profile compared to sodium valproate. ${ }^{32,33}$ The use of sodium valproate during the first trimester has been associated with major malformations and long-term complications in the form of developmental delay, lower intelligence quotient, and a higher risk of the development of autism spectrum disorder. ${ }^{32,34,35}$ It is worrisome that the majority of studied psychiatric patients in Saudi Arabia are receiving antipsychotic agents. Moreover, lithium is rarely used in women of childbearing age compared to valproate, which is linked to serious teratogenicity and other adverse effects.

In our study, $75.9 \%$ of elderly patients (older than 60 years) received antipsychotics, mainly second-generation drugs $(67.2 \%)$, whereas only $41 \%$ received antidepressants and $13.8 \%$ received mood stabilizers. This overuse of antipsychotics among the elderly, despite warnings against this practice, can be explained by their off-label uses for behavioral disturbances that are associated with some mental disorders, such as dementia. Many factors play a role in increasing the risks associated with antipsychotic use among the elderly, including age, associated medical problems, and drug interactions. Thus, the use of antipsychotics for this population should follow recommendations and guidelines. ${ }^{36}$

It is very interesting to note how in Saudi Arabia only a very small number of patients were prescribed antianxiety drugs. In contrast, alprazolam alone was the most prescribed psychiatric medication in the USA from 2005 to 2013, with $48,465,000$ total prescriptions in $2013 .{ }^{37}$ The greater legal restriction on psychotropic medication use within Saudi Arabian hospitals may be one of the reasons behind this trend, although it could also be that benzodiazepines may be overused in the private sector or as street drugs. ${ }^{38,39}$ Overall, the study findings indicate that the prescribing practices among psychiatric patients in Saudi Arabia do not conform well to international guidelines. ${ }^{40-42}$

\section{Conclusion}

Our study provides enough data to support and complement correct patient management and prescribing decisions for the 
use of psychotropic medications in both inpatient and outpatient settings. By investigating both previous and current treatments, we were able to produce an interesting, in-depth analysis that provides new point of view over a large and heterogeneous patient population from the five main regions of Saudi Arabia. Nevertheless, because of the use of convenience sampling, our results should be cautiously generalized to all psychiatric patients in Saudi Arabia. Another main limit is that the available data focus only on a small, 2-year time span (2012-2014); therefore, a more in-depth analysis of previous data may help to assess how prescriptions of psychotropic drugs have changed over the course of the last few years. This study may also help with assessing the burden of psychiatric illness in specific patient demographics and might be effectively used to strategically plan health resource allocation, generate new treatment hypotheses, or serve as a source of evidence that could further integrate other observational studies.

\section{Acknowledgment}

This project was funded by the National Plan for Science, Technology and Innovation (MAARIFAH), King Abdulaziz City for Science and Technology, Kingdom of Saudi Arabia, Award Number (MED3130).

\section{Disclosure}

The authors report no conflicts of interest in this work.

\section{References}

1. Davids E, Bunk C, Specka M, Gastpar M. Psychotropic drug prescription in a psychiatric university hospital in Germany. Prog Neuro-Psychopharmacology Biol Psychiatry. 2006;30(6):1109-1116.

2. Bowers L, Callaghan P, Clark N, Evers C. Comparisons of psychotropic drug prescribing patterns in acute psychiatric wards across Europe. Eur J Clin Pharmacol. 2004;60(1):29-35.

3. Kuroda N, Sun S, Lin CK, et al. Attitudes toward taking medication among outpatients with schizophrenia: cross-national comparison between Tokyo and Beijing. Environ Health Prev Med. 2008;13(5):288-295.

4. Deshmukh SA, Shaikh T, Shaikh E. Evaluation of psychotropic drugs use pattern among out patients attending psychiatry department at government medical college and hospital, Nagpur: a cross sectional study. Int J Pharm Bio Sci. 2012;3(3):428-436.

5. Yenilmez Ç, Güleç G, Büyükkınacı A, et al. Bir üniversite hastanesinde yatarak tedavi gören hastalarda çoklu ilaç kullanımı: Geriye dönük bir çalı̧̧ma [Polypharmacy among inpatients of a university psychiatry clinic: a retrospective study]. Dusunen Adam J Psychiatry Neurol Sci. 2012;25:43-50. Turkish.

6. Jordanova V, Maric NP, Alikaj V, et al. Prescribing practices in psychiatric hospitals in Eastern Europe. Eur Psychiatry. 2011;26(7):414-418.

7. Creed F, Black D, Anthony P, Osborn M, Thomas P, Tomenson B. Randomised controlled trial of day patient versus inpatient psychiatric treatment. BMJ. 1990;300(6731):1033-1037.

8. Leslie D, Rosenheck R. Shifting to outpatient care? Mental health care use and cost under private insurance. Am J Psychiatry. 1999;156(8): $1250-1257$.
9. Martin A, Leslie D. Psychiatric inpatient, outpatient, and medication utilization and costs among privately insured youths, 1997-2000. Am J Psychiatry. 2003;160(4):757-764.

10. Goldstein JM, Horgan CM. Inpatient and outpatient psychiatric services: substitutes or complements? Hosp Community Psychiatry. 1988;39(6):632-636.

11. Shahin SH, Daly EB. Knowledge, attitudes and beliefs about psychotropic medication among Saudi hospitalized psychiatric patients. Int $J$ Nurs Stud. 1999;36(1):51-55.

12. Alkhamis A. Health care system in Saudi Arabia: an overview. East Mediterr Heal J. 2012;18(10):1078-1079.

13. Al-Ghamdy YS, Qureshi NA, Abdelghadir MH, Al-Habeeb TA, Ahmad SA. Psychotropic drugs prescriptions in Al-Qassim Region, Saudi Arabia. East Mediterr Health J. 1999;5(1):27-34.

14. Courtney J. A Review of: "Kalyna Z Bezchlibnyk-Butler and Adil S. Varani (2004)." Child Neuropsychol. 2005;11:311-313.

15. Correll CU. Antipsychotic use in children and adolescents: minimizing adverse effects to maximize outcomes. $J$ Am Acad Child Adolesc Psychiatry. 2008;47(1):9-20.

16. Sutherland JE, Sutherland SJ, Hoehns JD. Achieving the best outcome in treatment of depression. J Farm Pract. 2003;52(3):201-209.

17. De Hert M, Detraux J, van Winkel R, Yu W, Correll CU. Metabolic and cardiovascular adverse effects associated with antipsychotic drugs. Nat Rev Endocrinol. 2011;8(2):114-126.

18. Newcomer JW. Second-generation (atypical) antipsychotics and metabolic effects: a comprehensive literature review. CNS Drugs. 2005;19(suppl 1):1-93.

19. Llorente MD, Urrutia V. Diabetes, psychiatric disorders, and the metabolic effects of antipsychotic medications. Clin Diabetes. 2006;24:18-24.

20. Hong $\mathrm{CH}, \mathrm{Oh} \mathrm{BH}$. Inappropriate prescribing in the elderly patients. J Korean Med Assoc. 2009;52(1):91-99.

21. Anonymous. Practice guideline for major depressive disorder in adults. Am J Psychiatry. 1993;150(4 Suppl):1-26.

22. Tungaraza TE, Farroq S. Clozapine prescribing in the UK: views and experience of consultant psychiatrists. Ther Adv Psychopharmacol. 2015;5(2):88-96.

23. Rothschild AJ. Challenges in the treatment of major depressive disorder with psychotic features. Schizophr Bull. 2013;39(4):787-796.

24. Spielmans GI. The promotion of olanzapine in primary care: an examination of internal industry documents. Soc Sci Med. 2009;27(69): 14-20.

25. Spielmans GI, Parry PI. From evidence-based medicine to marketingbased medicine: evidence from internal industry documents. J Bioethical Inq. 2010;7:13-29.

26. Spielsman IG. Atypical antipsychotics: overrated and overprescribed. Pharm J. 2015;294:7851.

27. Elaine H, John W. Metabolic screening after the American diabetes association's consensus statement on antipsychotic drugs and diabetes. Diabetes Care. 2009;32:1037-1042.

28. Review CE. First- and second-generation antipsychotics for children and young adults. Comp Eff Rev. 2012;39.

29. Leucht S, Corves C, Arbter D, Engel RR, Li C, Davis JM. Secondgeneration versus first-generation antipsychotic drugs for schizophrenia: a meta-analysis. Lancet. 2009;373(9657):31-41.

30. McCrea RL, Nazareth I, Evans SJW, et al. Lithium prescribing during pregnancy: a UK primary care database study. PLoS One. 2015;10(3):1-8.

31. Winser KL, Leckman-Westin E, Finnerty M, Essock SM. Valproate prescription prevalence among women of childbearing age. Psychiatr Serv. 2011;62(2):218-220.

32. Grover S, Avasthi A. Mood stabilizers in pregnancy and lactation. Indian J Psychiatry. 2015;57:S308-S323. Available from: http:// search.ebscohost.com/login.aspx?direct=true $\& \mathrm{db}=\mathrm{edb} \& \mathrm{AN}=108714$ 121\&site=eds-live. Accessed December 31, 2015.

33. Adedinsewo DA, Thurman DJ, Luo Y-H, Williamson RS, Odewole OA, Oakley GP Jr. Valproate prescriptions for nonepilepsy disorders in reproductive-age women. Birth Defects Res A Clin Mol Teratol. 2013; 97(6):403-408. 
34. Arkilo D, Vaou EO. Antiepileptic drug use during pregnancy: sodium valproate is associated with lower offspring IQ. Evid Based Nurs. 2015;18(4):109.

35. Singh S. Valproate use during pregnancy was linked to autism spectrum disorder and childhood autism in offspring. Ann Intern Med. 2013;159(4):JC13.

36. Gareri P, Segura-García C, Manfredi VGL, et al. Use of atypical antipsychotics in the elderly: a clinical review. Clin Interv Aging. 2014;9:1363-1373.

37. Fayek M, Flowers C, Signorelli D, Simpson G. Psychopharmacology: underuse of evidence-based treatments in psychiatry. Psychiatr Serv. 2003;54(11):1453-1454.

38. Hodge K, Jespersen S. Side-effects and treatment with clozapine: a comparison between the views of consumers and their clinicians. Int J Ment Health Nurs. 2008;17(1):2-8.
39. Al-Sabaie AS, Abdul-Rahim FA, Oni GA, Hawari RA. The use of sedative-hypnotic drugs in a university hospital in Saudi Arabia. Ann Saudi Med. 1994;14(5):379-382.

40. Yatham LN, Kennedy SH, Parikh SV, et al. Canadian Network for Mood and Anxiety Treatments (CANMAT) and International Society for Bipolar Disorders (ISBD) collaborative update of CANMAT guidelines for the management of patients with bipolar disorder: update 2013. Bipolar Disord. 2013;15(1):1-44.

41. Association AP. American Psychiatric Association Practice Guidelines for the Treatment of Psychiatric Disorders: Compendium 2006. Arlington, VA: American Psychiatric Association; 2006.

42. Taylor D, Paton C, Kapur S. The Maudsley Prescribing Guidelines in Psychiatry. Portsmouth: John Wiley \& Sons; 2015.
Neuropsychiatric Disease and Treatment

\section{Publish your work in this journal}

Neuropsychiatric Disease and Treatment is an international, peerreviewed journal of clinical therapeutics and pharmacology focusing on concise rapid reporting of clinical or pre-clinical studies on a range of neuropsychiatric and neurological disorders. This journal is indexed on PubMed Central, the 'PsycINFO' database and CAS,

\section{Dovepress}

and is the official journal of The International Neuropsychiatric Association (INA). The manuscript management system is completely online and includes a very quick and fair peer-review system, which is all easy to use. Visit http://www.dovepress.com/testimonials.php to read real quotes from published authors.

Submit your manuscript here: http://www.dovepress.com/neuropsychiatric-disease-and-treatment-journal 